\section{In vivo imaging of Sarcoptes scabiei infestation using line-field confocal optical coherence tomography}

\section{Dear Editor,}

Scabies remains a worldwide spread disease of considerable health and economic burden with repeated outbreaks in community settings. ${ }^{1}$ Scabies agent is the ectoparasite Sarcoptes scabiei var. hominis; this invisible mite causes a progressive rash, which can be misdiagnosed with eczema or pruritus sine materia. Diagnosis is based on characteristic clinical features (intense nightly itch, inflammatory excoriated papules in typical sites) and history of contact to infected patients. According to the new International Alliance for the Control of Scabies (IACS) classification, the identification of mites, eggs or faecal pellets through skin scraping, high magnification devices or dermoscopy counts as diagnostic criteria for confirmed scabies. ${ }^{1}$ The typical dermoscopic sign of scabies infestation is the so-called 'delta', 'kite' or 'triangle', representing the anterior body of the adult female mite at the end of the burrow ${ }^{2}$ (Fig. 1e). Reflectance confocal microscopy (RCM)3 and optical coherence tomography (OCT) were used in the detection of mite infestations (scabies, demodex) ${ }^{4,5}$ and their therapeutic monitoring. ${ }^{6,7}$ Line-field confocal optical coherence tomography (LC-OCT, DAMAE Medical ${ }^{\circledR}$, Paris, France) is new imaging technique that can also be used to visualize mites and related skin changes. LC-OCT is based on a two-beam interference microscope with line illumination and line detection using a broadband spatially coherent light source and a line-scan camera. ${ }^{8}$ It has three imaging modalities: en-coupe (vertical), en-face and $3 \mathrm{D}$, providing a cellular resolution $(1.1 \times 1.3 \mu \mathrm{m})$.

A patient (male, 50 years old) with suspect symptoms and skin rash was examined with LC-OCT (DAMAE Medical, Paris, France). Mites were also identified by high magnification digital dermoscopy (Fotofinder ${ }^{\circledR}$, Bad Birnbach, Germany). In LCOCT en-face images, S. scabiei was clearly identifiable as an ovoid structure with a thin, corrugated exoskeleton and triangular spicules on the dorsal surface, localized right below the

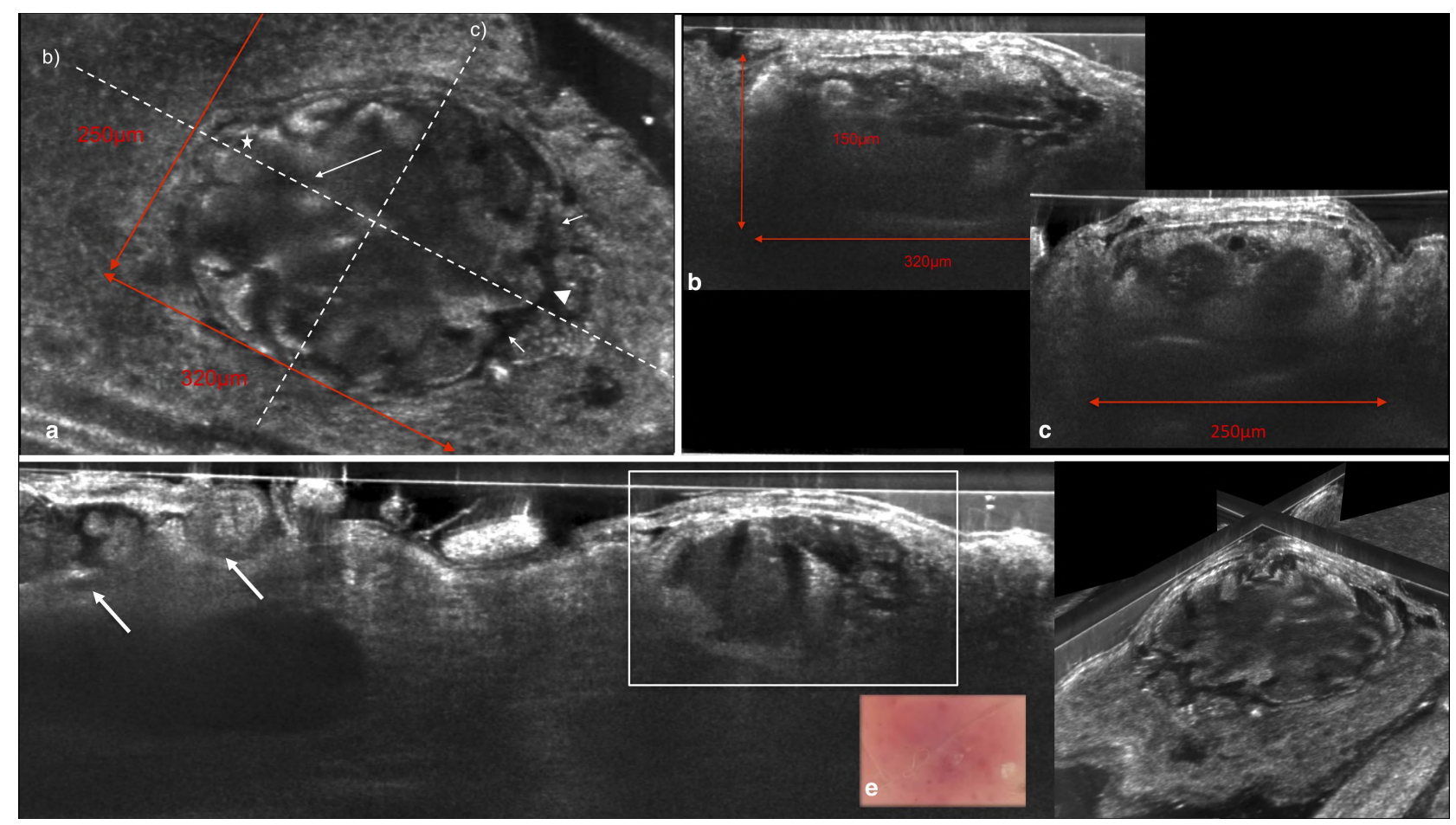

Figure 1 a) In en-face LC-OCT images, S. scabiei can be identified as a round structure in a burrow with a thin, corrugated exoskeleton and triangular spicules on the dorsal surface. Two pairs of hyperreflective coned structures (short arrows) corresponding to legs surround the polygonal head (triangle). At the opposite side, a triangular terminal anus (star) is visible and in the centre, a central, hyporeflective, convoluted gut (long arrow) with skybala and hyperreflective glands can be seen. 1b,c) Vertical LC-OCT images corresponding to the b) and c) axes in the $1 \mathrm{~A}$ en-face image 1d) Vertical LC-OCT image with a thick hyperkeratotic burrow surrounding the mite inside the epidermis. The ovoid burrow behind the mite contains the hyperreflective skybala and eggs. 1e) Dermoscopy of the scabies mite with the socalled 'delta' or 'kite' or 'triangle' sign 1f) 3D reconstruction of the hyperreflective burrow containing the mite body. 
stratum corneum in a burrow (Fig. 1a). Two pairs of hyperreflective coned structures corresponding to legs surround the polygonal, even brighter head (Fig. 1a, triangle). At the caudal extremity, a triangular terminal anus is visible (Fig. 1a, star). Internal organs are also hinted at a central, hyporeflective, convoluted gut with skybala (Fig. 1, long arrow) and hyperreflective (probably ovary and vitelline) glands.

In LC-OCT vertical images (Fig. 1b,c,d) and 3D reconstruction (Fig. 1f), the exact location of the mite inside the epidermis and Stratum corneum can be observed. A thick hyperkeratotic scale corresponding to the burrow covers it. The optical density of the mite is comparable to the surrounding tissue; however, the hyporeflective burrow lumen and the scaly hyperreflective burrow wall build a fringe, which delimitates the mite body. An ovoid or longitudinal burrow can be also visualized behind the mite, depending on the scan direction. Skybala and eggs can also be identified as roundish, mainly hyperreflective structures.

The in vivo observation of scabies mites in their human hosts allows a quick diagnosis at bedside and provides interesting information about the biology of the living mites. Not only OCT and RCM but also LC-OCT permits fast, painless, real-time imaging of mites, their morphology and their skin location, with hints to their internal structures. ${ }^{3,9}$ LC-OCT has a better resolution of conventional OCT, but still lower than RCM; this is however compensated by the possibility of combining en-face and vertical views and obtaining $3 \mathrm{D}$ reconstructions at different angles. Burrows, eggs and skybala can also be visualized easily. The high resolution and penetration depth of LC-OCT combined with the possibility of acquiring vertical, en-face and $3 \mathrm{D}$ images provides more details compared to dermoscopy and other diagnostic techniques, which may possibly reduce diagnostic pitfalls. Moreover, exact special correlations and measurements are possible. This allows an immediate diagnosis of scabies infestation as well as non-invasive therapeutic monitoring.

We believe LC-OCT is a non-invasive diagnostic tool with high potential in dermatology, with a broad field of application and likely to be applied in the future not only to skin cancer ${ }^{10}$ but also in skin infestations.

\section{Acknowledgement}

The authors would like to thank DAMAE Medical for making the LC-OCT system available for this study.

\section{Conflicts of interest}

The authors declare no conflict of interest.

$$
\begin{array}{r}
\text { C. Ruini, }{ }^{1,2,{ }^{*}, \dagger} \text { S. Schuh, }{ }^{3, \dagger} \text { G. Pellacani, }{ }^{2} \text { L. French, }{ }^{1} \mathrm{~J}^{-} \\
\text {Welzel, }{ }^{3, \dagger} \text { E. Sattler }
\end{array}
$$

Dermatology and Allergy, University Hospital Augsburg, Augsburg, Germany

${ }^{*}$ Correspondence: C Ruini. E-mail: cristel.ruini@med.uni-muenchen.de ‘Both first and senior authors contributed equally.

\section{References}

1 Engelman D, Yoshizumi J, Hay RJ et al. The 2020 International Alliance for the Control of Scabies Consensus Criteria for the diagnosis of scabies. Br J Dermatol 2020. https://doi.org/10.1111/bjd.18943

2 Argenziano G, Fabbrocini G, Delfino M. Epiluminescence microscopy. A new approach to in vivo detection of Sarcoptes scabiei. Arch Dermatol 1997; 133: 751-3.

3 Longo C, Bassoli S, Monari P, Seidenari S, Pellacani G. Reflectance-mode confocal microscopy for the in vivo detection of Sarcoptes scabiei. Arch Dermatol 2005; 141: 1336.

4 Ruini C, Sattler E, Hartmann D, Reinholz M, Ruzicka T, von Braunmuhl T. Monitoring structural changes in Demodex mites under topical Ivermectin in rosacea by means of reflectance confocal microscopy: a case series. J Eur Acad Dermatol Venereol 2016; 31: e299-e301.

5 Micali G, Lacarrubba F, Verzi AE, Chosidow O, Schwartz RA. Scabies: Advances in Noninvasive Diagnosis. PLoS Negl Trop Dis 2016; 10: e0004691.

6 Sattler EC, Hoffmann VS, Ruzicka T, Braunmuhl TV, Berking C. Reflectance confocal microscopy for monitoring the density of Demodex mites in patients with rosacea before and after treatment. Br J Dermatol 2015; 173: 69-75.

7 Welzel JSS. Nichtinvasive Diagnostik in der Dermatologie. J Dtsch Dermatol Ges 2017; 15: 999-1017.

8 Ogien J, Levecq O, Azimani H, Dubois A. Dual-mode line-field confocal optical coherence tomography for ultrahigh-resolution vertical and horizontal section imaging of human skin in vivo. Biomed Opt Express 2020; 11: 1327-35.

9 Welzel J, Lankenau E, Birngruber R, Engelhardt R. Optical coherence tomography of the skin. Curr Probl Dermatol 1998; 26: 27-37.

10 Dubois A, Levecq O, Azimani $\mathrm{H}$ et al. Line-field confocal optical coherence tomography for high-resolution noninvasive imaging of skin tumors. J Biomed Opt 2018; 23: 1-9.

DOI: $10.1111 / \mathrm{jdv} .16671$ 